Research Article

\title{
Deep Learning-Based Ultrasound Imaging Diagnosis for Gonadotropin-Releasing Hormone Agonists Treatment of Central Precocious Puberty
}

\author{
Ruifang Qi ${ }^{1},{ }^{1}$ Kun Yang $\mathbb{D}^{2},{ }^{2}$ and Rongmin Li ${ }^{1}$ \\ ${ }^{1}$ Department of Endocrinology, Baoding Children's Hospital, Baoding 071000, Hebei, China \\ ${ }^{2}$ Child Health Section, Baoding Children's Hospital, Baoding 071000, Hebei, China \\ Correspondence should be addressed to Kun Yang; f12024104@st.sandau.edu.cn
}

Received 12 July 2021; Revised 2 August 2021; Accepted 11 August 2021; Published 1 September 2021

Academic Editor: Gustavo Ramirez

Copyright (c) 2021 Ruifang Qi et al. This is an open access article distributed under the Creative Commons Attribution License, which permits unrestricted use, distribution, and reproduction in any medium, provided the original work is properly cited.

To explore the adoption of ultrasound imaging diagnosis based on deep learning of convolutional neural networks (CNNs) in the treatment of central precocious puberty (CPP) by gonadotropin-releasing hormone agonists ( $\mathrm{GnRHa}$ ), ultrasound imaging based on CNN was utilized to treat CPP. The bone age, uterine and ovarian volume, and breast development of incomplete precocious puberty (IPP) group and CPP group were observed and recorded. The peak values of luteinizing hormone (LH) and folliclestimulating hormone (FSH) were measured. The uterine and ovarian volume before and after GnRHa treatment of CPP were compared. The results showed that the bone age $(9.03 \pm 1.07)$, uterine volume $(2.37 \pm 1.52)$, ovarian volume $(2.36 \pm 0.82 \mathrm{~mL})$, and breast development of the CPP group were considerably higher in contrast to the IPP group and control group $(P<0.05)$. The LH peak $(11.97 \pm 5.63)$ and FSH peak $(12.89 \pm 3.15)$ of the CPP group were substantially higher relative to the IPP group $(P<0.05)$. The uterine volume $(1.06 \pm 0.42)$ and ovarian volume $(1.12 \pm 0.49)$ after treatment were inferior to those before treatment $(P<0.05)$. In short, ultrasound images based on deep learning could diagnose precocious puberty, which could also provide a certain basis for GnRHa treatment of CPP, as well as an important basis for clinical diagnosis and treatment of precocious puberty.

\section{Introduction}

With the rapid development of social economy, food safety issues, environmental issues, family education issues, and mental and psychological issues have gradually become prominent, which begin to affect the physical and mental development of children, and the incidence of precocious puberty has increased notably in recent years [1]. Precocious puberty is an abnormality in the time of puberty, which refers to secondary sexual characteristics and internal and external genital development before the age of 8 in girls and 9 in boys $[2,3]$. According to reports, the prevalence of precocious puberty in children in China is about $0.47 \%$ [4]. According to whether the hypothalamic-pituitary-gonadal axis function is activated, precocious puberty is classified as peripheral precocious puberty and CPP. The most common type of precocious puberty in girls is CPP [5]. In addition,
CPP girls are too early at menarche, which may lead to psychological and behavioral abnormalities, and may cause social psychological behavior problems. Convolutional neural network $(\mathrm{CNN})$ is a feed-forward neural network that can effectively reduce the complexity of feedback neural networks. It can be used to identify some distorted and undeformed two-dimensional graphics such as displacement and zoom. It is one of the representative algorithms of deep learning. The wide application of deep learning such as $\mathrm{CNN}$ in medical image classification can provide convenience for researchers based on traditional research methods. Neural network recognition technology has begun to attract attention from all walks of life and is widely used in image segmentation. Neural network has a huge number of connections, making it easy to introduce spatial information, and can better solve the problems of unevenness and noise in image recognition. The first $\mathrm{CNN}$ is a time delay 
network proposed by foreign scholars and experts, which is a CNN for speech recognition problems. Secondly, translation invariant artificial neural networks have been successively proposed and applied to medical image detection and recognition. Later, with the development of deep learning theory and the updating of numerical computing equipment, the representation learning capabilities of CNNs began to receive widespread attention from all walks of life.

At present, GnRHa is a specific drug for the treatment of CPP. GnRHa reduces the sensitivity of $\mathrm{GnRH}$ receptors to natural GnRHa and inhibits HPGA function by competitively binding to $\mathrm{GnRH}$ receptors on the pituitary surface. In turn, it inhibits gonadal development and delays epiphyseal fusion $[6,7]$. It can also be seen in clinical practice that only some IPP with advanced sex characteristics are not accompanied by advanced bone age. In recent years, it has been found that some children with IPP can be converted to CPP. Thus, the diagnosis of CPP should be differentiated from IPP $[8,9]$. The GnRH provocation test is taken as the gold standard for the diagnosis of CPP and IPP, but the provocation test is time-consuming and less sensitive. Therefore, the clinical approach combined with ultrasound can improve the diagnostic efficiency and is simple, noninvasive, and reproducible $[10,11]$. In recent years, the development of deep learning has brought new opportunities for medical image analysis. At present, many models of deep learning have been applied in medical ultrasound. Abdelhafiz et al. [12] utilized CNN to detect lesions in mammography and obtain accurate lesion locations. However, the adoption of deep learning ultrasound images to the diagnosis of precocious puberty in girls is relatively rare, and more research is required.

In summary, ultrasound technology can be adopted to diagnose precocious puberty, and CPP can be treated by injection of GnRHa. Therefore, the ultrasound images based on the CNN deep learning model were utilized to observe and measure the girls' bone age, uterus and ovarian volume, and the hypoechoic area under the breast. Combined with the GnRH provocation test, the peak values of serum LH and $\mathrm{FSH}$ in girls were measured, to preliminary diagnose CPP and IPP. Children with CPP were treated with GnRHa injection, and their uterus and ovarian volume before and after treatment were measured and compared, to provide a theoretical basis for clinical diagnosis and treatment of precocious puberty.

\section{Materials and Methods}

2.1. Research Subjects. The precocious puberty girls who were admitted to the hospital from February 2018 to January 2020 were taken as the research subjects, among which 26 cases of girls were diagnosed with CPP, the age range was $5-8$ years, and the average age was $6.73 \pm 1.32$ years. There were 26 cases of girls who were diagnosed as IPP, and their age range was $4-8$ years, with an average age of $6.64 \pm 1.53$ years. A total of 26 healthy and normal girls were selected as the controls, the age range was $5-8$ years, and the average age was $6.18 \pm 1.15$ years. The study had been approved by the Ethics Committee of Hospital, and the patients and their families understood the situation of the study and signed the informed consent forms.

CPP inclusion criteria were as follows. I: early appearance of secondary sexual characteristics, that is, girls presented with secondary sexual characteristics such as breast development, appearance of pubic hair, and increased vaginal discharge before the age of 8 ; II: the bone age was 1 year older than actual age or even more; III: patients with complete preoperative ultrasound examination data; IV: GnRH provocation test: serum samples of girls were collected at $0,30,60$, and 90 minutes after intravenous injection of Gonadorelin (GnRH); the serum LH and FSH values were measured; immunochemiluminometric assay (ICMA) was adopted to measure LH peak value (no less than $5.0 \mathrm{IU} / \mathrm{L}$ ), and $\mathrm{LH}$ peak value/FSH peak value (over 0.6); then CPP can be diagnosed; V: enlarged gonads: the ovarian volume over $1 \mathrm{~mL}$ was seen under B-ultrasound, and multiple follicles with diameter over $0.4 \mathrm{~cm}$ were seen; VI: patients who had been in good health and could normally cooperate with the trial for treatment.

Inclusion criteria of IPP were as follows. I: secondary sexual characteristics appeared early: the girl developed simple breast development, menarche, or early pubic hair development before the age of 8; II: no obvious pigmentation in areola and no coffee and milk spots in skin during physical examination; III: GnRH stimulation test: the serum LH and FSH values were measured at $0,30,60$, and 90 min after intravenous injection of $\mathrm{GnRH}$. The peak value of $\mathrm{LH}$ was no more than 3.0 IU/L, and the peak value of LH/FSH was less than 0.6 measured by ICMA. IV: the bone age was not advanced.

Exclusion criteria of CPP and IPP were as follows. I: patients with thyroid gland dysfunction and other endocrine diseases; II: patients with other diseases causing secondary precocious puberty; III: long-term intake of exogenous glucocorticoids; IV: patients with serious diseases of the heart, liver, kidney, or hematopoietic system; V: patients who dropped out of the experiment due to their own reasons.

2.2. Deep Learning Model: CNN. Through the training and mining of large amounts of data, deep learning can sum up hidden useful information from it, to achieve the purpose of correct prediction and analysis of unknown data; thus it is widely adopted in speech recognition, image recognition, natural language processing, and other fields. CNN is a discriminative deep learning model, used for image signal and voice signal processing, and has shown excellent results in image and voice applications.

Under normal circumstances, CNNs mainly include convolutional layers, pooling layers, and fully connected layers, which optimize the training and updates the parameters through the loss function. Convolutional layer is the most critical basic structure in $\mathrm{CNN}$, which mainly implements the network convolution operation on the data. If the input single sample data vector is $x_{i}^{a}=\left[x_{1}, x_{2}, \ldots x_{m}\right]$, then the output signal $C$ calculated by the convolution layer is as follows: 


$$
C_{i}^{t, n}=f\left(d_{n}^{t}+\sum_{s=1}^{S} h_{s}^{n} \cdot x_{i+s-1}^{\mathrm{an}}\right) .
$$

In (1), $C$ is the output signal, $X_{i}^{a}$ is the single sample data vector, $t$ is the index of the layer, $f$ is the activation function, which is used to introduce nonlinear processing to the layer, $d$ is the deviation term of the $\mathrm{n}$-th feature map, $S$ represents the size of the convolution kernel, and $h s$ represents the weight value of the $n$-th feature map and the $s$-th filter. The main function of the convolutional layer is to extract multiple features of a local area through different filters, to achieve the effect of layer-by-layer feature abstraction and data dimensionality reduction.

The pooling layer is also called the subsampling layer, which implements compression and filtering of the feature map by subsampling the input data to reduce information redundancy. Pooling is generally classified as maximum pooling and average pooling. The maximum pooling calculation method is (2), and the average pooling calculation method is (3).

$$
\begin{aligned}
& P_{i}^{t, n}=\max _{k \in K} C_{i \times L+k}^{t, n}, \\
& P_{i}^{t, n}=\frac{1}{|K|} \sum_{k \in K} C_{i \times L+k}^{t, n} .
\end{aligned}
$$

In (2), $P$ represents the maximum pooling, $C$ is the output signal, $K$ represents the size of the pooling window, and $L$ represents the pooling step size. In (3), $P$ represents average pooling, $K$ represents the size of the pooling window, and $L$ represents the pooling step size. The pooling layer can reduce the complexity of the model and reduce the burden of the classifier.

The fully connected layer connects each neuron in the output layer and the upper input layer to achieve feature integration. The corresponding calculation method is as follows:

$$
Q_{i}^{t, n}=f\left(d_{n}^{t}+g_{i}^{t, n} \cdot P_{i}^{t, n}\right) .
$$

In (4), $Q$ represents the fully connected layer, $f$ represents the activation function, $g$ represents the weight value of the fully connected layer, and $d$ represents the deviation term of the $n$-th feature map.

2.3. Ultrasound Examination. LOGIQ E9 convex array probe (GE company) was utilized for uterine and ovarian examination, and the frequency was $7.5 \mathrm{MHz}$. During the examination, the girls were moderately filled with water in their bladder and lied on the examination bed in supine position, and their lower abdomen was fully exposed. Then, the uterus in cross section was examined, and the transverse diameter of the uterus was measured under the uterine angle plane. Longitudinal-section examination of the uterus was done; simultaneously the uterine floor, uterine cavity, and intrauterine incision firm were shown. The length of the uterus (the outer margin of the uterus to the internal cervical orifice) and the anteroposterior diameter (the maximum longitude perpendicular to the length of the uterus) were measured. The length diameter and anteroposterior diameter of the ovary were measured on the maximum section of the ovary's long axis. The probe rotated $90^{\circ}$ on the maximum section of the ovary's long axis. The maximum transverse diameter of ovary was measured. Uterine volume $(\mathrm{mL})=($ long diameter $\times$ anteroposterior diameter$\times$ transverse diameter $) \times 0.52$; ovarian volume $(\mathrm{mL})=($ long diameter $\times$ transverse diameter $\times$ anteroposterior diameter) $\times 0.52$, and the number of follicles whose diameter was greater than $0.4 \mathrm{~cm}$ was recorded.

GE company LOGIQ L9 linear array probe was utilized for breast ultrasound examination with a frequency of 9.5 MHz. The probe was placed horizontally above the nipple to display the inferior echoic area below the nipple, and the length diameter and thickness of the inferior echoic area were recorded.

2.4. Hormone Detection. For all precocious puberty girls, $2 \mathrm{~mL}$ of venous blood was drawn in the morning in a quiet state, and GnRH was then injected intravenously for $2.5 \mu \mathrm{g} /$ $\mathrm{kg}$ per time with a maximum dose of $100 \mu \mathrm{g}$. Blood samples were collected at $30 \mathrm{~min}, 60 \mathrm{~min}$, and $90 \mathrm{~min}$ after injection, and $\mathrm{LH}$ value and FSH value were measured.

2.5. Detection of the Skeletal Age. Bone age examination was conducted within one week of GnRH stimulation test. X-ray films of left wrist and knuckle were taken for all girls, and bone age was measured according to G-P method. The DR800 machine of GE company was adopted. During the shooting, the palm of the left hand was close to the box, the five fingers were naturally separated, and the center of the ball tube was aimed at the third metacarpal head.

2.6. GnRHa Treatment. Uterine volume and ovarian volume were tested after the fourth injection of GnRHa for all girls with CPP. The uterine volume and ovarian volume were compared before and after treatment.

2.7. Statistical Methods. Data processing was analyzed by SPSS 19.0 version statistical software, measurement data were represented by mean \pm standard deviation $(\overline{\mathrm{x}} \pm s)$, and counting data were represented by percentage (\%). Pairwise comparison was performed via analysis of variance. The difference was statistically significant at $P<0.05$.

\section{Results}

3.1. Descriptive Statistics of Basic Patient Data. The age range of 26 cases in the CPP group was 5-8 years, the average age was $6.73 \pm 1.32$ years, and the bone age was $9.03 \pm 1.07$. The age range of the IPP group was $4-8$ years, with an average age of $6.64 \pm 1.53$ years and a bone age of $7.35 \pm 1.21$. The age range of the control group was 5-8 years, with an average age of $6.69 \pm 1.47$ years and a bone age of $7.12 \pm 1.13$. In Figure 1, the average age of the CPP group, IPP group, and control group had no notable difference $(P>0.05)$. However, the 


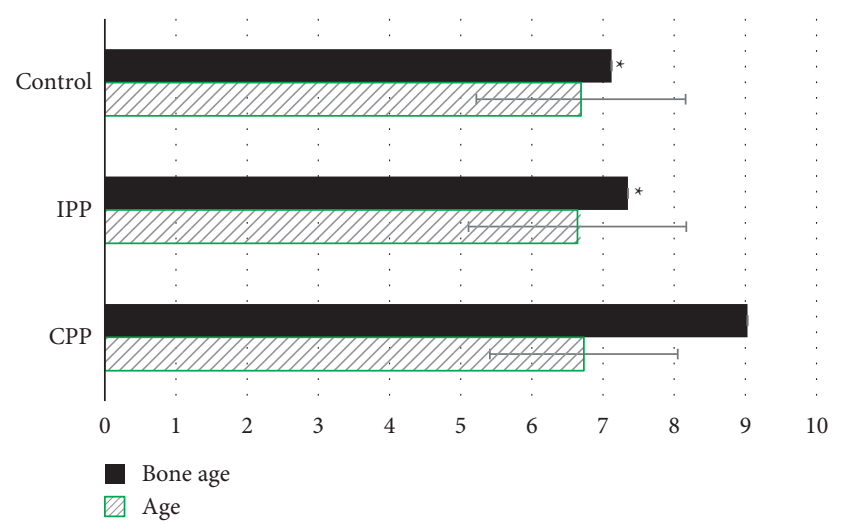

FIgURE 1: Comparison of average age and bone age of girls in CPP, IPP, and control groups. Note. * indicates that the difference was substantial relative to the CPP group $(P<0.05)$.

bone age of the CPP group was notably greater in contrast to the IPP group and the controls, and the difference was very considerable $(P<0.05)$. There was no remarkable difference in bone age between the IPP group and the controls $(P>0.05)$.

As shown in Figure 2, it was a bone age image of a girl aged 7 years and 3 months with CPP, and the bone age was 10 years.

3.2. Hormone Detection Results. The basic value of LH and the basic value of FSH of the CPP group and IPP group were detected. The basic value of $\mathrm{LH}$ of the CPP group was $1.76 \pm 1.49 \mathrm{IU} / \mathrm{L}$, and the basic value of FSH was $3.68 \pm 1.97 \mathrm{IU} / \mathrm{L}$; the basic value of LH of the IPP group was $0.41 \pm 0.30 \mathrm{IU} / \mathrm{L}$, and the basic value of FSH was $2.57 \pm 1.12 \mathrm{IU} / \mathrm{L}$. In Figure 3, the basic values of LH and FSH of the CPP group were substantially superior to those of the IPP group, with evident difference $(P<0.05)$.

In the $\mathrm{GnRH}$ provocation test, the $\mathrm{LH}$ peak value of the CPP group was $11.97 \pm 5.63 \mathrm{IU} / \mathrm{L}$, and the $\mathrm{FSH}$ peak value was $12.89 \pm 3.15 \mathrm{IU} / \mathrm{L}$; the $\mathrm{LH}$ peak value of the IPP group was $2.69 \pm 1.13 \mathrm{IU} / \mathrm{L}$, and the FSH peak value was $5.76 \pm 5.21 \mathrm{IU} / \mathrm{L}$. In Figure 4, the LH peak and FSH peak of the CPP group were higher in contrast to those of the IPP group, with notable difference $(P<0.05)$. Moreover, the LH peak difference was more obvious than the FSH peak. The $\mathrm{LH}$ peak/FSH peak of the CPP group was $0.94 \pm 0.37 \mathrm{IU} / \mathrm{L}$, and the LH peak/FSH peak of the IPP group was $0.43 \pm 0.13 \mathrm{IU} / \mathrm{L}$. In Figure 5, the peak LH/peak FSH of the CPP group was considerably greater relative to the peak LH/ $\mathrm{FSH}$ of the IPP group, and the difference was very obvious $(P<0.05)$.

\subsection{Ultrasound Inspection Results Based on Deep Learning.} The uterus and ovaries of 26 girls in the CPP group, IPP group, and normal control group were observed by ultrasound examination. A standard measurement of the transverse diameter $\times$ the upper and lower diameter $\times$ the front and rear diameter of the uterus and ovary of the girl was done. Then, the volume of the uterus and ovaries was calculated. The results showed that the uterine volume of girls in the CPP group was $2.37 \pm 1.52 \mathrm{~mL}$, and the uterine volume of girls in the IPP group was $1.32 \pm 1.03 \mathrm{~mL}$. The uterine volume of normal girls in the control group was $0.81 \pm 0.34 \mathrm{~mL}$. In Figure 6 , the uterine volume of girls in the CPP group was greatly larger in contrast to the IPP group and controls, with considerable difference $(P<0.05)$. Moreover, the uterine volume of girls in the IPP group was also higher in contrast to normal control group $(P<0.05)$.

The ovarian volume of girls in the CPP group was $2.36 \pm 0.82 \mathrm{~mL}$, the ovarian volume of girls in the IPP group was $1.39 \pm 0.53 \mathrm{~mL}$, and that in controls was $0.91 \pm 0.32 \mathrm{~mL}$. In Figure 7, the ovarian volume of girls in the CPP group was notably greater versus IPP group and controls $(P<0.05)$. The ovarian volume of girls in the IPP group was also higher versus the controls $(P<0.05)$.

The transverse diameter of the hypoechoic area under the right breast in the CPP group was $27.65 \pm 9.24 \mathrm{~mm}$, and the thickness was $8.75 \pm 3.12 \mathrm{~mm}$. The transverse diameter of the hypoechoic area under the left breast was $28.39 \pm 9.35 \mathrm{~mm}$, and the thickness was $8.93 \pm 3.06 \mathrm{~mm}$. The transverse diameter of the hypoechoic area under the right breast in the IPP group was $18.76 \pm 9.32 \mathrm{~mm}$, and the thickness was $6.52 \pm 3.21 \mathrm{~mm}$. The transverse diameter of the hypoechoic area under the left breast was $18.03 \pm 9.25 \mathrm{~mm}$, and the thickness was $6.46 \pm 2.89 \mathrm{~mm}$. Figure 8 shows that the left and right breast transverse diameters in the CPP group were greatly larger versus those in the IPP group $(P<0.05)$. In Figure 9, the thickness of the left and right breasts in the CPP group was obviously greater versus that in the IPP group $(P<0.05)$.

3.4. Uterus and Ovarian Volume in CPP Group before and after GnRHa Treatment. GnRHa was injected into all children in the CPP group; after the fourth injection, the uterine volume of the children in the CPP group was $1.16 \pm 0.42 \mathrm{~mL}$ and the ovarian volume was $1.12 \pm 0.49 \mathrm{~mL}$. In Figure 10, after GnRHa treatment in the CPP group, the uterus and ovarian volume were evidently smaller than before treatment, with statistical differences $(P<0.05)$.

\section{Discussion}

Due to the increase in the pulse release amplitude of GnRH, CPP upregulates the secretion levels of gonadotropins and sex hormones in children, leading to accelerated development of children [13]. IPP is only manifested as part of the characteristic vocalization in advance but not accompanied by advanced bone age. The child can recover on its own within a period of time without treatment. Since it is found that part of IPP will be converted into CPP [14], the differential diagnosis of CPP and IPP is imperative before treatment. Ultrasound examination can accurately reflect the morphological structure of the reproductive organs in the child and can improve the accuracy of diagnosis after combined with the deep learning model in ultrasound images $[15,16]$. The CNN deep learning model can improve the efficiency of network computing, and the efficient 


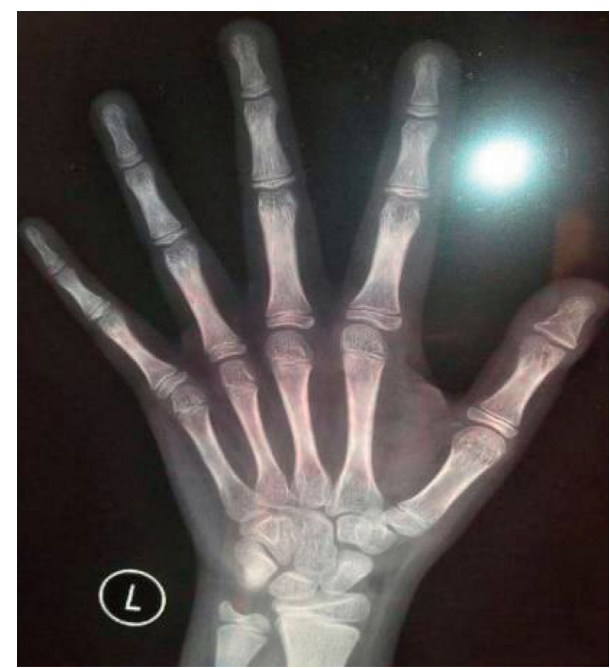

FIGURE 2: Image of bone age of a girl aged 7 years and 3 months.

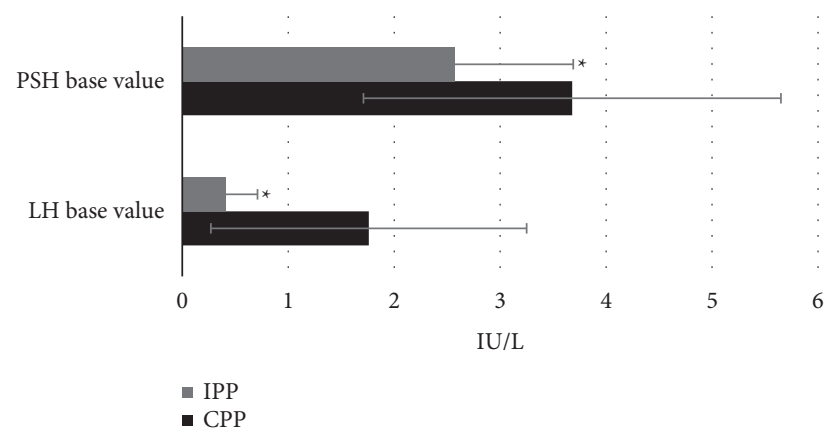

FIGURE 3: Comparison of basic values of LH and FSH between CPP group and IPP group. Note. * means that the difference was considerable versus CPP group $(P<0.05)$.

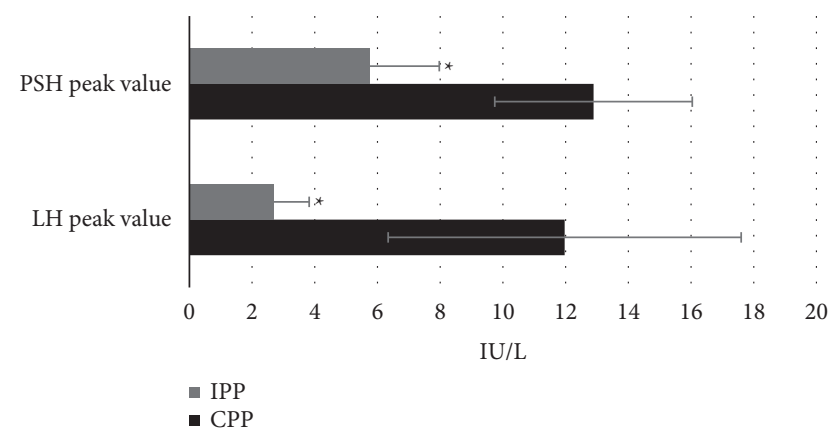

FIGURE 4: Comparison of LH and FSH peaks between CPP group and IPP group. Note. * suggests that the difference was considerable in contrast to the CPP group $(P<0.05)$.

processing of visual data plays an important role in ultrasound image diagnosis [17]. GnRHa is a relatively safe treatment drug for precocious puberty in the current extensive clinical application [18]. At present, there are relatively few studies on applying deep learning ultrasound images to the diagnosis of precocious puberty in female children, and more research is needed. Therefore, ultrasound images based on the CNN deep learning model was adopted

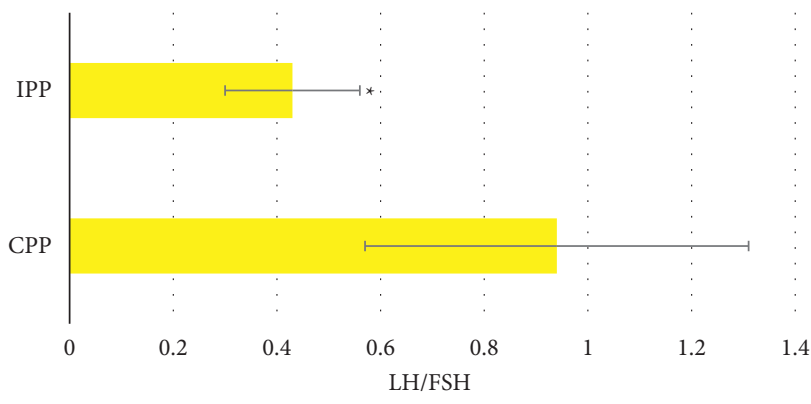

FIGURE 5: Comparison of LH peak/FSH peak between CPP group and IPP group. Note. * suggests that the difference was substantial relative to the CPP group $(P<0.05)$.

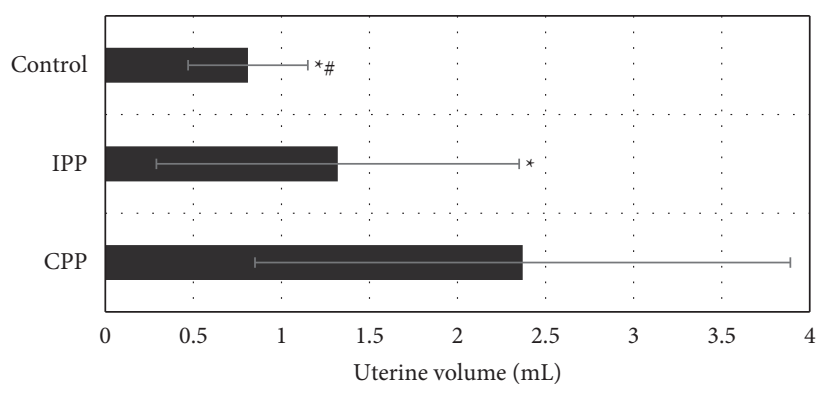

Figure 6: Comparison of uterine volume of three groups of girls. Note. $*$ and \# indicate that the difference was remarkable relative to the CPP group and IPP group, respectively (both $P<0.05$ ).

to observe and measure the girl's bone age, uterus and ovarian volume, and hypoechoic area under the breast. Then, CPP and IPP were initially diagnosed, and children with CPP was treated by injection of GnRHa. The uterus and ovarian volume of children before and after treatment were compared, so as to provide an important basis for clinical diagnosis and treatment of precocious puberty.

Compared with girls of average age, the bone age can accurately reflect the development of children. It was found that the bone age of the CPP group $(9.03 \pm 1.07)$ was evidently greater versus IPP group $(7.35 \pm 1.21)$ and controls $(7.12 \pm 1.13)(P<0.05)$. It meant that the bone development of children with CPP was obviously advanced, which was consistent with the research results of Lee et al. [19]. In the GnRH challenge test, the LH peak $(11.97 \pm 5.63)$ and FSH peak $(12.89 \pm 3.15)$ of the CPP group were greatly higher relative to the IPP group, whose LH peak was $2.69 \pm 1.13$ and FSH peak was $5.67 \pm 2.21$. The developmental status of the uterus, ovaries, and breasts of the girls with CPP and IPP was confirmed by ultrasound examination. The results showed that the uterine volume $(2.37 \pm 1.52 \mathrm{~mL})$ of the CPP group was highly greater than uterine volume $(1.32 \pm 1.03 \mathrm{~mL})$ of the IPP group and control group $(0.81 \pm 0.34 \mathrm{~mL})$. The ovarian volume $(2.36 \pm 0.82 \mathrm{~mL})$ was also larger than the ovarian volume $(1.39 \pm 0.53 \mathrm{~mL})$ of the IPP group and control group $(0.91 \pm 0.32 \mathrm{~mL})$. The transverse diameter and thickness of the bilateral breasts in the CPP group were greater in contrast to the IPP group $(P<0.05)$. Through the injection of GnRHa in the CPP group, the results showed that the uterine volume $(1.06 \pm 0.42)$ and ovarian volume 

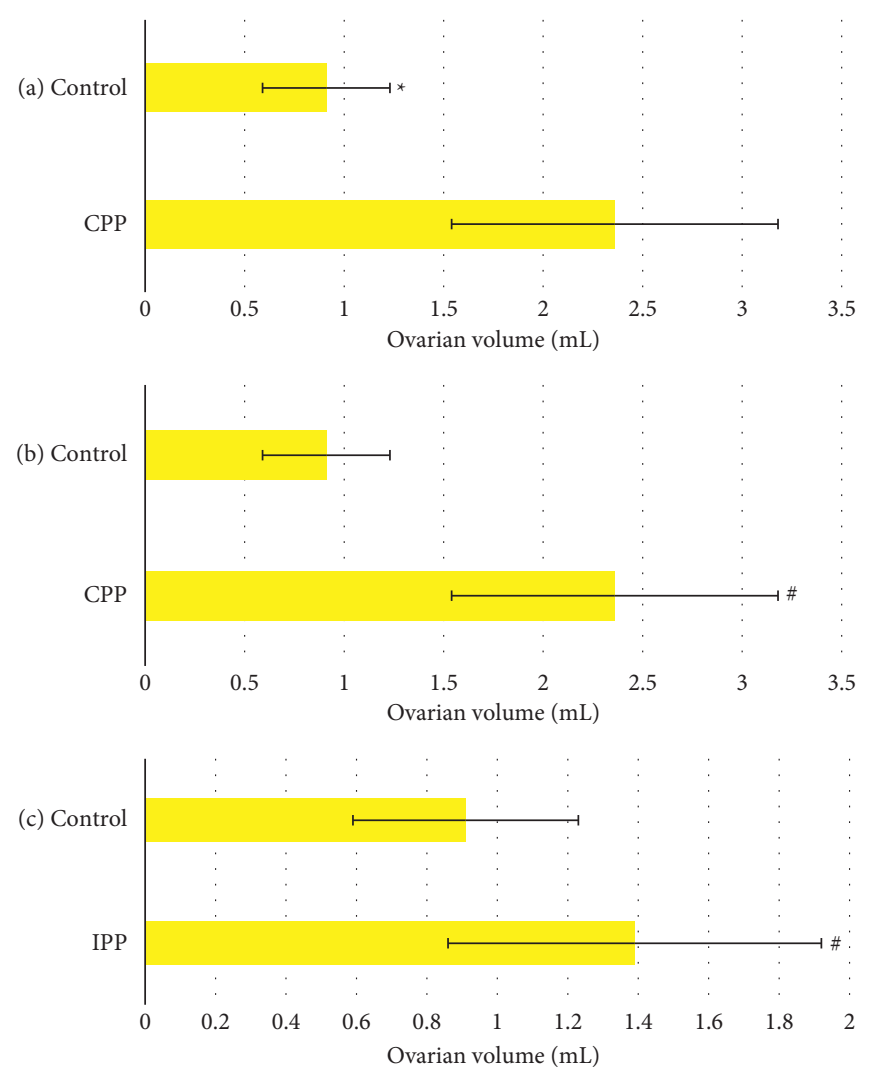

FIgURE 7: Comparison of ovarian volume of three groups of girls. Note. * and \# mean that the difference was considerable when compared to the CPP group and IPP group, respectively (both $P<0.05$ ).

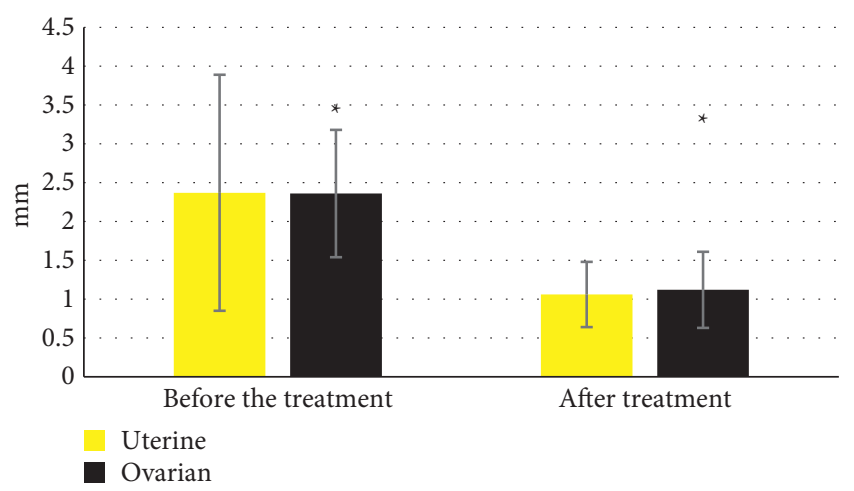

FIGURE 8: Comparison of measured values of bilateral breast transverse diameters between two groups of girls. Note. * indicates substantial differences relative to the CPP group $(P<0.05)$.

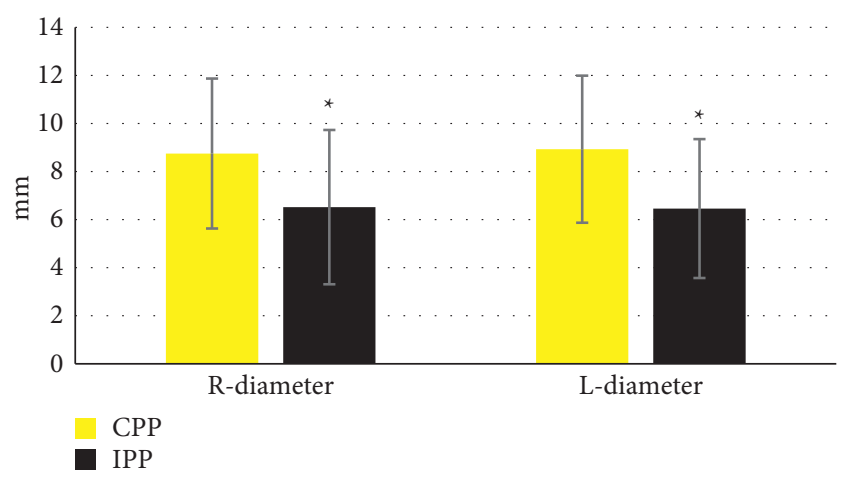

Figure 9: Comparison of the measured values of bilateral breast thickness between two groups of girls. Note. * suggests highly notable differences when compared to the CPP group $(P<0.05)$. 


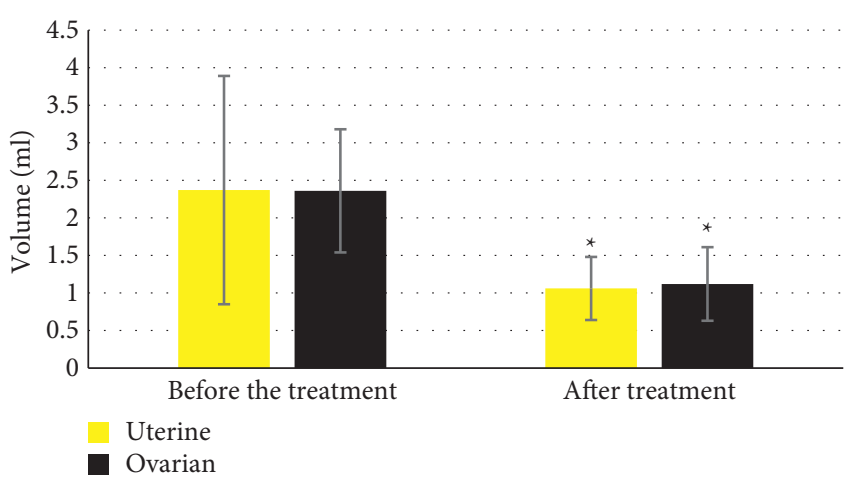

FIGURE 10: Comparison of girl's uterus and ovarian volume before and after treatment in the CPP group. Note. * indicates that the difference was statistically substantial relative to that before the treatment $(P<0.05)$.

$(1.12 \pm 0.49)$ after treatment were obviously inferior to those before treatment, with obvious differences $(P<0.05)$. This result was consistent with the results of Xue et al. [20], indicating that GnRHa had a certain effect in the treatment of CPP

\section{Conclusion}

By adopting ultrasound images based on the CNN deep learning model, the girl's bone age, uterus and ovarian volume, and hypoechoic area under the breast were observed and measured. Combined with the GnRH provocation test, the LH peak and FSH peak of the girl were measured, to initially diagnose CPP and IPP. GnRHa injection was performed on children with CPP, and the uterus and ovarian volume of the children before and after treatment were compared. The results revealed that ultrasound images based on deep learning can diagnose precocious puberty, which can provide a basis for GnRHa treatment of CPP, and provide an important basis for clinical diagnosis and treatment of precocious puberty. Due to the small sample size of patients selected in this study, it may cause certain deviations in the results. In the follow-up studies, it should consider increasing the scope of selection of the number of patients and further explore the adoption of ultrasound diagnosis in GnRHa treatment of CPP. In short, ultrasound images based on deep learning can initially diagnose precocious puberty, which can provide a certain basis for the follow-up GnRHa treatment of CPP, as well as an important basis for clinical diagnosis and treatment of precocious puberty.

\section{Data Availability}

The data used to support the findings of this study are available from the corresponding author upon request.

\section{Conflicts of Interest}

The authors declare no conflicts of interest.

\section{References}

[1] R. S. Aguirre and E. A. Eugster, "Central precocious puberty: from genetics to treatment," Best Practice \& Research Clinical Endocrinology \& Metabolism, vol. 32, no. 4, pp. 343-354, Aug 2018.

[2] A. C. Latronico, V. N. Brito, and J.-C. Carel, "Causes, diagnosis, and treatment of central precocious puberty," The Lancet Diabetes \& Endocrinology, vol. 4, no. 3, pp. 265-274, Mar 2016.

[3] V. N. Brito, A. M. Spinola-Castro, C. Kochi, C. Kopacek, P. C. A. d. Silva, and G. Guerra-Júnior, "Central precocious puberty: revisiting the diagnosis and therapeutic management," Archives of Endocrinology and Metabolism, vol. 60, no. 2, pp. 163-172, Apr 2016.

[4] A. Persichetti, E. Di Stasio, R. Guglielmi et al., "Predictive value of malignancy of thyroid nodule ultrasound classification systems: a prospective study," Journal of Clinical Endocrinology \& Metabolism, vol. 103, no. 4, pp. 1359-1368, Apr 2018.

[5] S. Cantas-Orsdemir and E. A. Eugster, "Update on central precocious puberty: from etiologies to outcomes," Expert Review of Endocrinology and Metabolism, vol. 14, no. 2, pp. 123-130, Mar 2019.

[6] A. P. M. Canton, C. E. Seraphim, V. N. Brito, and A. C. Latronico, "Pioneering studies on monogenic central precocious puberty," Archives of Endocrinology and Metabolism, vol. 63, no. 4, pp. 438-444, Aug 222019.

[7] S. Leka-Emiri, G. P. Chrousos, and C. Kanaka-Gantenbein, "The mystery of puberty initiation: genetics and epigenetics of idiopathic central precocious puberty (ICPP)," Journal of Endocrinological Investigation, vol. 40, no. 8, pp. 789-802, Aug 2017.

[8] A. Bereket, "A critical appraisal of the effect of gonadotropinreleasing hormon analog treatment on adult height of girls with central precocious puberty," Journal of Clinical Research in Pediatric Endocrinology, vol. 9, no. 2, pp. 33-48, Dec 30 2017.

[9] Y. J. Kim, A. Kwon, M. K. Jung et al., "Incidence and prevalence of central precocious puberty in korea: an epidemiologic study based on a national database," The Journal of Pediatrics, vol. 208, pp. 221-228, May 2019.

[10] J. Gao, Q. Jiang, Q. Jiang, B. Zhou, and D. Chen, "Convolutional neural networks for computer-aided detection or diagnosis in medical image analysis: an overview," Mathematical Biosciences and Engineering, vol. 16, no. 6, pp. 6536-6561, Jul 152019.

[11] S. M. Anwar, M. Majid, A. Qayyum, M. Awais, M. Alnowami, and M. K. Khan, "Medical image analysis using convolutional neural networks: a review," Journal of Medical Systems, vol. 42, no. 11, p. 226, Oct 82018.

[12] D. Abdelhafiz, C. Yang, R. Ammar, and S. Nabavi, "Deep convolutional neural networks for mammography: advances, challenges and applications," BMC Bioinformatics, vol. 20, no. S11, p. 281, Jun 62019.

[13] V. S. L. Williams, A. M. Soliman, A. M. Barrett, and K. O. Klein, "Review and evaluation of patient-centered psychosocial assessments for children with central precocious puberty or early puberty," Journal of Pediatric Endocrinology \& Metabolism, vol. 31, no. 5, pp. 485-495, Apr 252018.

[14] A. D. Buluş, A. Așci, P. Erkekoglu, A. Balci, N. Andiran, and B. Koçer-Gümüşel, "The evaluation of possible role of endocrine disruptors in central and peripheral precocious 
puberty," Toxicology Mechanisms and Methods, vol. 26, no. 7, pp. 493-500, Sep 2016.

[15] H. Dobnig and K. Amrein, "Value of monopolar and bipolar radiofrequency ablation for the treatment of benign thyroid nodules," Best Practice \& Research Clinical Endocrinology \& Metabolism, vol. 33, no. 4, Article ID 101283, Aug 2019.

[16] T. Durá-Travé, M. Ortega Pérez, L. Ahmed-Mohamed, P. Moreno-González, M. J. Chueca Guindulain, and S. Berrade-Zubiri, "Central precocious puberty in girls: diagnostic study and auxological response to triptorelin treatment," Endocrinología, Diabetes y Nutrición (English ed.), vol. 66, no. 7, pp. 410-416, Aug-Sep 2019.

[17] H. Chereda, A. Bleckmann, F. Kramer, A. Leha, and T. Beissbarth, "Utilizing molecular network information via graph convolutional neural networks to predict metastatic event in breast cancer," Studies in Health Technology and Informatics, vol. 267, pp. 181-186, Sep 32019.

[18] F. N. Tessler, W. D. Middleton, E. G. Grant, and J. K. Hoang, "Re: ACR thyroid imaging, reporting and data system (TIRADS): white paper of the ACR TI-RADS committee," Journal of the American College of Radiology, vol. 15, no. 3, pp. 381-382, Mar 2018.

[19] H. S. Lee, J. S. Yoon, K. J. Park, and J. S. Hwang, "Increased final adult height by gonadotropin-releasing hormone agonist in girls with idiopathic central precocious puberty," PloS One, vol. 13, no. 8, Article ID e0201906, Aug 222018.

[20] Y. Xue, P. Wang, S. Q. Wang, and Y. Q. Gao, "The clinical effects of gonadotropin-releasing hormone agonists for the treatment of children patients with central precocious puberty," European Review for Medical and Pharmacological Sciences, vol. 22, no. 15, pp. 5014-5017, Aug 2018. 